\title{
A Polytopic Approach for the Nonlinear Unknown Input Functional Observers Design: Application to a Quadrotor Aerial Robots Landing
}

\author{
Souad Bezzaoucha ${ }^{1}$, Holger Voos ${ }^{1}$ and Mohamed Darouach ${ }^{2}$
}

\begin{abstract}
This paper considers a method of designing functional observers for continuous time nonlinear systems subject to unknown inputs. The proposed approach consists in rewriting the nonlinear model in a polytopic form (also known as multiple or Takagi-Sugeno models) applying the Sector Nonlinearity Transformation (SNT) and then apply the proposed algorithm for the observer design. Sufficient conditions for the asymptotic stability of the estimation error are given in terms of linear matrix inequalities $(\mathcal{L} \mathcal{M} \mathcal{I} s)$ and rank conditions. An application to a quadrotor aerial robots landing is then presented.
\end{abstract}

\footnotetext{
${ }^{1}$ Souad Bezzaoucha and Holger Voos are with the Interdisciplinary Centre for Security, Reliability and Trust (SnT), Automatic Control Research Group, University of Luxembourg, Campus Kirchberg, 6 rue Coudenhove-Kalergi L-1359, Luxembourg souad. bezzaoucha, holger.vooseuni.lu

${ }^{2}$ Mohamed Darouach is with the Research Center for Automatic Control of Nancy (CRAN), Université de Lorraine, IUT de Longwy, 186 rue de Lorraine, 54400 Cosnes et Romain, France mohamed.darouach@univ-lorraine.fr
} 